\title{
Prognostic value of albumin-red cell distribution width score in patients with severe community-acquired pneumonia
}

\author{
Li Chen, Xiao Ye Lu, Chang Qing Zhu \\ Department of Emergency Medicine, Renji Hospital, School of Medicine, Shanghai Jiaotong University, Shanghai 200127, China \\ Contributions: (I) Conception and design: L Chen; (II) Administrative support: None; (III) Provision of study materials or patients: All authors; (IV) \\ Collection and assembly of data: All authors; (V) Data analysis and interpretation: All authors; (VI) Manuscript writing: All authors; (VII) Final \\ approval of manuscript: All authors. \\ Correspondence to: Chang Qing Zhu. Department of Emergency Medicine, Renji Hospital, School of Medicine, Shanghai Jiaotong University, 160 \\ Pujian Road, Shanghai 200127, China. Email: zhucq1965@126.com.
}

\begin{abstract}
Background: It has been recently reported that albumin (ALB) and red cell distribution width (RDW) are correlated with the prognosis of community-acquired pneumonia, while their role in severe communityacquired pneumonia (sCAP) is unknown. This study aimed to investigate the correlation between the ALBRDW score and 90-day mortality of sCAP patients.

Methods: A total of 179 sCAP patients admitted to our hospital between January 1, 2014 and June 30, 2019 were enrolled in this study. Data of patient characteristics and laboratory data were obtained and CURB-65 was calculated. The ALB-RDW score was calculated based on serum ALB and RDW. Receiver operating characteristic (ROC) curve and the value of area under the curve (AUC) were used to evaluate the association between ALB-RDW score and 90-day mortality of sCAP patients.

Results: Compared with survivors, age, white blood cell counts, neutrophil counts, RDW, D-dimer, serum creatinine, lactic dehydrogenase, CURB-65 score, ALB-RDW high score ratio, invasive ventilation and septic shock were higher in non-survivors, while ALB and hemoglobin (HB) were lower. Multivariate logistic regression analysis identified that high age, serum creatinine, ALB-RDW score and invasive ventilation were the independent risk factors of 90-day mortality $(\mathrm{P}<0.05)$. Moreover, the AUC of ALB-RDW score was 0.742 [95\% confidence interval (CI): 0.667-0.817], which was superior to CURB-65 score 0.725 (95\% CI: 0.651-0.798). The AUC increased to 0.798 (95\% CI: 0.733-0.862) when combined with CURB-65 score.

Conclusions: High age, serum creatinine, ALB-RDW score and invasive ventilation were the independent risk factors of 90-day mortality of sCAP patients. In addition, the correlation between ALB-RDW and 90-day mortality was even stronger when combined with CURB-65 score.
\end{abstract}

Keywords: Severe community-acquired pneumonia (sCAP); albumin (ALB); red cell distribution width (RDW); mortality; CURB-65

Submitted Aug 31, 2019. Accepted for publication Feb 14, 2020.

doi: $10.21037 /$ apm.2020.04.22

View this article at: http://dx.doi.org/10.21037/apm.2020.04.22

\section{Introduction}

Severe community-acquired pneumonia (sCAP) is caused by the aggravation of lung tissue inflammation and it is associated with high morbidity and mortality. The mortality in hospitalized sCAP patients ranges from $25 \%$ to $50 \%(1,2)$. Despite recent advance in antibiotic treatment of CAP, the mortality remains high, and which adds heavy burden to the health care system
(3-5). Disease severity assessment and early diagnosis of CAP may improve the prognosis, and reduce the cost of medical care. Many clinicians pay attention to the assessment of the severity of CAP. Until now, several scoring systems have been developed to predict prognosis. For example, the pneumonia severity index (PSI), the CURB-65, new sepsis definition (sepsis-3), the national early warning score (NEWS), EXPANDED curb- 
65 and Expanded A-DROP Score (6-11). However, most of them are limited in emergencies department (ED). Among these scoring systems, the CURB-65 score is a relatively simple assessment tool that focuses on only five factors that associated with mortality, therefore we selected CURB-65 score as the criterion in this study.

Several risk factors have been studied alone or in combination with illness severity scores in attempting to predict the mortality of community-acquired pneumonia. Among these risk factors, serum albumin (ALB) and red cell distribution width (RDW) are commonly used laboratory parameters in hospitalized patients. Serum ALB is a routine marker which reflects both nutritional status and systemic inflammation. Recently, it has been showed that low serum ALB level is associated with mortality of community-acquired pneumonia (12-17). RDW measures the size variability of erythrocytes, and it is widely used in the diagnosis of anemia. Many studies reported that high $\mathrm{RDW}$ level is associated with poor short-term and long-term prognosis of CAP patients (18-22). However, little is known about the association of ALB and RDW in 90-day mortality of sCAP patients. The ALB-RDW score consists of the serum $\mathrm{ALB}$ and RDW that can reflect inflammatory conditions. In this study we evaluated the correlation between ALB-RDW score and 90-day mortality of sCAP patients.

\section{Methods}

\section{Patients}

Patients admitted to the emergency medical ward and emergency intensive care unit of Renji Hospital, School of Medicine, Shanghai Jiaotong University (Shanghai, China) due to sCAP between January 1, 2014 and June 30, 2019 were retrospectively enrolled in the study. Data of patient characteristics and laboratory data were collected in a web-based electronic medical recording system during hospitalization. Those who were transferred to other hospitals or automatically signed out of the hospital before treatment completed were excluded. Patient information and their medical insurance numbers were maintained encrypted. The study was approved by the Ethics Committee of Renji Hospital [No. 2016-080k(b)].

\section{Definitions}

According to the 2007 Infectious Diseases Society of America/American Thoracic Society (IDSA/ATS) guidelines (23), sCAP was defined as the presence of two major criteria (receipt of invasive mechanical ventilation and septic shock with the need for vasopressors), or presence of three minor criteria (respiratory rate $\geq 30$ breaths $/ \mathrm{min}$, $\mathrm{PaO}_{2} / \mathrm{FiO}_{2} \leq 250 \mathrm{mmHg}$, multilobar infiltrates, confusion and/or disorientation), uremia [blood urea nitrogen (BUN) level $\geq 20 \mathrm{mg} / \mathrm{dL}$ ], leucopenia [white blood cell (WBC) count $<4 \times 10^{9}$ cells $/ \mathrm{L}$ ], thrombocytopenia (platelet count $<100 \times 10^{9}$ platelets/L), hypothermia (core temperature $\leq 36{ }^{\circ} \mathrm{C}$ ), hypotension [systolic blood pressure (SBP) $\leq 90 \mathrm{mmHg}$; requiring aggressive fluid resuscitation]. CURB-65 score (a severity score based on mental confusion, BUN levels $\geq 20 \mathrm{mg} / \mathrm{dL}$, respiratory rate $\geq 30 / \mathrm{min}$, SBP $<90 \mathrm{mmHg}$ or diastolic blood pressure $\leq 60 \mathrm{mmHg}$ and age $\geq 65$ years) was used to assess the severity of pneumonia. The primary endpoint was 90 -day mortality in our study.

\section{Data compilation}

Age, sex, co-morbidity (diabetes mellitus, hepatopathy, chronic kidney disease, chronic obstructive pulmonary disease, coronary artery disease), smoking, mental confusion , invasive ventilation, septic shock, white cell counts, neutrophil counts, lymphocyte counts, platelet counts, RDW, hemoglobin level, ALB level, lactic acid level et al. were collected by electronic medical record system. ALB-RDW score was calculated according to the receiver operating characteristic (ROC) curve.

\section{Statistical analysis}

Normally distributed continuous variables were compared by Student's $t$-test and presented as mean \pm standard deviation. Nonnormally distributed continuous variables were expressed as median (interquartile range) and compared by Mann-Whitney $U$ test. Categorical variables were expressed as absolute numbers and relative frequencies and compared using the Chi-square test. Forward logistic regression was applied to determine the association between various hematological indices and 90-day mortality, while controlling for potential confounding. ROC curves were constructed to evaluate the prediction ability of ALB-RDW and CURB-65 score in 90-day mortality. All tests were two-tailed and significance was set at 0.05 . All statistical procedures were performed with SPSS 22.0.

\section{Results}

A total of 179 patients were enrolled in this study. Baseline 
Table 1 Baseline characteristics

\begin{tabular}{|c|c|c|c|c|}
\hline Characteristics & Overall $(\mathrm{N}=179)$ & Survivors (N=106) & Non-survivors ( $\mathrm{N}=73$ ) & $\mathrm{P}$ \\
\hline Male gender, n (\%) & $119(66.5)$ & $68(64.2)$ & $51(69.9)$ & 0.52 \\
\hline Smoking, n (\%) & $58(32.4)$ & $34(32.1)$ & $24(32.9)$ & 1 \\
\hline Invasive ventilation, n (\%) & $32(17.9)$ & $8(7.5)$ & $24(32.9)$ & $<0.001$ \\
\hline \multicolumn{5}{|l|}{ Co-morbidity, n (\%) } \\
\hline $\mathrm{DM}$ & $33(18.4)$ & $24(22.6)$ & $9(12.3)$ & 0.116 \\
\hline Hepatopathy & $15(8.4)$ & $12(11.3)$ & $3(4.1)$ & 0.105 \\
\hline CKD & $12(6.7)$ & $4(3.8)$ & $8(11.0)$ & 0.072 \\
\hline \multicolumn{5}{|l|}{ Laboratory data } \\
\hline WBC $\left(\times 10^{9} / L\right)$ & $9.36(6.06-13.93)$ & $8.12(4.81-12.63)$ & $10.70(6.73-17.82)$ & 0.001 \\
\hline Neutrophil $\left(\times 10^{9} / L\right)$ & $7.86(4.83-12.26)$ & $6.69(4.16-10.92)$ & $9.76(6.17-16.45)$ & $<0.001$ \\
\hline Lymphocyte (×10\%/L) & $0.73(0.49-1.07)$ & $0.77(0.53-1.12)$ & $0.68(0.45-1.03)$ & 0.143 \\
\hline Platelet $\left(\times 10^{9} / \mathrm{L}\right)$ & 188 (124.00-264.00) & $194(127.00-282.50)$ & $187(124.00-235.00)$ & 0.277 \\
\hline RDW (\%) & $13.50(12.80-14.70)$ & $13.10(12.60-13.85)$ & $14.00(13.20-15.40)$ & $<0.001$ \\
\hline $\mathrm{CRP}(\mathrm{mg} / \mathrm{L})$ & $116.00(40.30-173.81)$ & 122.09 (50.05-171.74) & $116.00(37.05-182.00)$ & 0.908 \\
\hline D-dimer $(\mu \mathrm{g} / \mathrm{mL})$ & $1.20(0.68-2.62)$ & $1.04(0.67-2.45)$ & $2.00(0.85-3.93)$ & 0.007 \\
\hline $\mathrm{HB}(\mathrm{g} / \mathrm{L})$, mean $\pm \mathrm{SD}$ & $113.30 \pm 24.39$ & $118.60 \pm 20.74$ & $107.51 \pm 25.72$ & $<0.001$ \\
\hline CURB-65 & $2[1-2]$ & 1 [0-2] & 2 [2-3] & $<0.001$ \\
\hline ALB-RDW score $(0 / 1 / 2)$ & $87 / 67 / 25$ & $70 / 31 / 5$ & $17 / 36 / 20$ & $<0.001$ \\
\hline
\end{tabular}

Data are presented as $\mathrm{n}$ (\%) or median (interquartile range), unless otherwise indicated. DM, diabetes mellitus; CKD, chronic kidney disease; CAD, coronary artery disease; COPD, chronic obstructive pulmonary disease; WBC, white blood cell; RDW, red cell distribution width; CRP, C-reactive protein; GPT, glutamic-pyruvic transaminase; LDH, lactic dehydrogenase; ALB, albumin; HB, hemoglobin; ALBRDW score, albumin-red cell distribution width score.

characteristics including co-morbidity and laboratory data were listed in Table 1 . The overall median age was 65 years (53-79 years). The median age of non-survivors was 76 years (62-82 years), which is much higher than 63 years (51-71 years) of survivors $(\mathrm{P}<0.001)$. Invasive ventilation and septic shock were more common in the non-survivors
$(\mathrm{P}<0.05)$. There was no difference in sex smoking and comorbidity between them.

Inflammatory factors, hemoglobin, ALB and lactic acid were associated with mortality of patients that suffer from inflammatory diseases (24-26). We found that ALB $(26.52 \pm 4.99$ vs. $30.01 \pm 4.78 \mathrm{~g} / \mathrm{L}, \mathrm{P}<0.001)$ and hemoglobin 
Table 2 Multivariate regression results of 90-day mortality for sCAP

\begin{tabular}{|c|c|c|c|c|}
\hline Risk factors & OR & \multicolumn{2}{|c|}{$95 \% \mathrm{Cl}$} & $P$ \\
\hline Age (years) & 0.943 & 0.911 & 0.977 & 0.001 \\
\hline Invasive ventilation & 5.719 & 1.796 & 18.209 & 0.003 \\
\hline Septic shock & 1.847 & 0.491 & 6.955 & 0.364 \\
\hline Neutrophil $\left(\times 10^{9} / \mathrm{L}\right)$ & 0.859 & 0.599 & 1.233 & 0.411 \\
\hline $\mathrm{D}$-dimer $(\mu \mathrm{g} / \mathrm{mL})$ & 0.891 & 0.778 & 1.021 & 0.098 \\
\hline Creatinine ( $\mu \mathrm{mol} / \mathrm{L})$ & 0.993 & 0.987 & 0.999 & 0.031 \\
\hline LDH (U/L) & 0.999 & 0.998 & 1.001 & 0.239 \\
\hline ALB (g/L) & 0.976 & 0.87 & 1.093 & 0.670 \\
\hline ALB-RDW score $(0 / 1 / 2)$ & 0.299 & 0.107 & 0.836 & 0.021 \\
\hline CURB-65 & 1.349 & 0.816 & 2.232 & 0.243 \\
\hline
\end{tabular}

SCAP, severe community-acquired pneumonia; OR odds ratio; Cl confidence interval; LDH, lactic dehydrogenase; HB, hemoglobin; RDW, red cell distribution width; ALB albumin; ALB-RDW score, albumin-red cell distribution width score.

level $(107.51 \pm 25.72$ vs. $118.60 \pm 20.74 \mathrm{~g} / \mathrm{L}, \mathrm{P}<0.001)$ are lower in non-survivors than that of the survivors. White cells, Neutrophil, D-dimer, serum creatinine, lactic dehydrogenase and RDW were higher in non-survivors than these in survivors. There was no statistically significant difference in lymphocyte counts, platelet counts, C-reactive protein (CRP) and lactate between them. According to the ROC curve, the cutoff values of serum ALB and RDW were set at $27.75 \mathrm{~g} / \mathrm{L}$ and $14.75 \%$, respectively. The ALBRDW score was classified into three groups based on each cutoff value of serum ALB and RDW. Patients with both hypoalbuminemia $(\leq 27.75 \mathrm{~g} / \mathrm{L})$ and high $\mathrm{RDW}(>14.75 \%)$ was defined as score 2. Patients with either of two abnormities was defined as score 1 , patients with neither hypoalbuminemia nor high RDW was defined as score 0 . Seventeen (23.3\%), 36 (49.3\%), 20 (27.4\%) of non-survivors had an ALB-RDW score of 0,1 , and 2, respectively, based on the system of ALB-RDW score. Seventy (66.0\%) of survivors had an ALB-RDW score of 0 , and 31 (29.2\%) of them had an ALB-RDW score of 1, 5 (4.7\%) of them had an ALB-RDW score of 2. ALB-RDW score was higher in non-survivors compared with survivors $(\mathrm{P}<0.001)$.

The results of the multivariate analysis were listed in Table 2. The results showed that ALB-RDW score [odds ratio $(\mathrm{OR})=0.299,95 \%$ confidence interval $(\mathrm{CI})$ : 0.107-0.836], age (OR $=0.943,95 \%$ CI: $0.911-0.977)$, invasive ventilation ( $\mathrm{OR}=5.719,95 \% \mathrm{CI}: 1.796-18.209)$, serum creatinine (OR $=0.993,95 \%$ CI: $0.987-0.999)$ were independent risk factors of 90-day mortality. We further calculated the area under the curves (AUCs) of CURB-65 score, ALB-RDW score and the combination of CURB65 and ALB-RDW score. As shown in Figure 1, the AUC of ALB-RDW score was 0.742 (95\% CI: $0.667-0.817$ ), which was higher than CURB-65 score 0.725 (95\% CI: 0.651-0.798). When ALB-RDW score was combined with CURB-65, the AUC value was increased to 0.798 (95\% CI: 0.733-0.862), which was higher than either CURB-65 score or ALB-RDW score alone $(\mathrm{P}<0.05)$.

\section{Discussion}

In this study, by analysis of 179 sCAP patients, we found that non-survivors were relatively older. They had higher value in total WBC count, neutrophil count, serum creatinine, lactic dehydrogenase, RDW, D-dimer, and they had lower level of ALB and hemoglobin. While the survivors had lower invasive ventilation, septic shock, CURB-65 score and ALB-RDW score. Multivariate 


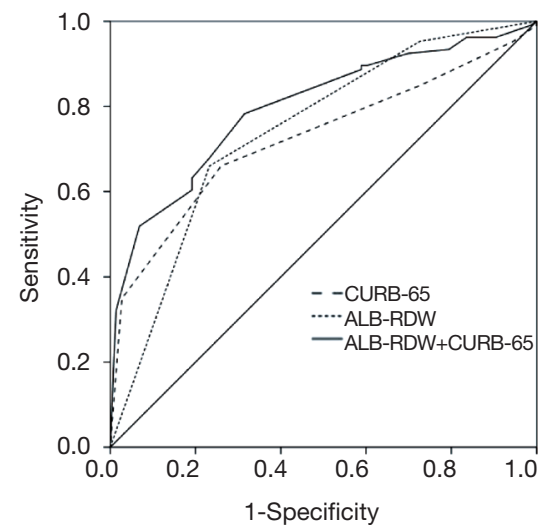

\begin{tabular}{lcc}
\hline Variables & AUC $(95 \% \mathrm{Cl})$ & $\mathrm{P}$ \\
\hline ALB-RDW+CURB-65 & $0.798(0.733-0.862)$ & \\
CURB-65 & $0.725(0.651-0.798)$ & 0.012 \\
ALB+RDW & $0.742(0.667-0.817)$ & 0.002 \\
\hline
\end{tabular}

Figure 1 The areas under the curves (AUC) of ALB-RDW score, CURB-65 score, and CURB-65 combined with ALB-RDW score. ALB-RDW score, albumin-red cell distribution width score.

analysis showed that high age, serum creatinine, invasive ventilation and ALB-RDW score were independently associated with 90-day mortality in hospitalized sCAP patients. In addition, we demonstrated that ALB-RDW combined with CURB-65 were strongly associated with 90-day mortality, the association was stronger than CURB65 alone or ALB-RDW alone.

ALB is a medium size molecule that is synthesized in the liver, it plays an important role in inflammatory response and oxidative stress. It has been showed that ALB is a prognostic marker and associated with mortality of CAP patients (12-17). A prospective study from 1995 to 2011 in Barcelona showed that hypoalbuminemia $(<30 \mathrm{~g} / \mathrm{L})$ significantly increased the ability of prediction of CURB-65/PSI scores in CAP patients (12). In 2015, Lee et al. determined that ALB $<33 \mathrm{~g} / \mathrm{L}$ was a risk factor of 30-day mortality of CAP patients especially when combined with PSI (13). In addition, a retrospective study about 797 CAP patients in 2016 suggested that ALB level was a risk factors of 30-day mortality of CAP patients, the correlation was even stronger when combined with CURB-65 or PSI (14). Holter and colleague found that low serum ALB level was an independent risk factor of CAP caused death, the death risk increases by $25 \%$ for each $5 \mathrm{~g} / \mathrm{L}$ decrease in the patients' serum ALB level (15). A study about 1,834
CAP patients at Kurashili Central Hospital reported that low ALB level was correlated with a worse prognosis (16). Another study (17) revealed that ALB, compared with PSI, was stronger risk factor of 30-day mortality of patients with pneumonia occurring outside hospital. In our study, ALB was selected as one of the scoring criteria and the cutoff value of ALB was $27.75 \mathrm{~g} / \mathrm{L}$. However, the mechanisms underlying the hypoalbuminemia and the mortality of CAP are unclear. May be factors that could affect ALB synthesis, distribution, or both of them need to be considered.

RDW is a clinical index that is routinely examined as part of the complete blood count. Many studies have revealed that RDW is a risk factor of mortality in some conditions. In our study, the cutoff value of RDW is $14.75 \%$ that is similar to other previous studies. A study of Braun et al. (18) about young hospitalized CAP patients showed that RDW $(>14.5 \%)$ was an independent risk factor of 90-day mortality. The study by Lee et al. in 2013 showed that RDW (>15.2\%) was associated with 30-day mortality and improved the prognostic performance of PSI and CURB-65 (19). In another study of community-acquired pneumonia in elderly people, RDW $(>15 \%)$ as a prognostic marker was unrelated with WBC count or hemoglobin levels and associated with 90-day mortality (20). Moreover, according to a long-term study in 2015 (21) showed that RDW (>14\%) improved the accuracy of PSI and CURB-65 at 2 and 3 years, significantly increasing their ROC curves. A study about adult CAP patients from 2006 to 2012 revealed that high RDW (>15\%) was associated with increased risk of 30-day mortality (22). Although it is not known about the mechanisms behind that how RDW is associated with the prognosis of diseases, systemic inflammation and oxidative stress have been suggested to be involved in (27).

ALB-RDW score is based on ALB and RDW that are not one of the values used to calculate the CURB-65. ALB and RDW can be obtained from blood routine examination and easy to be used without any further calculation needed. It does not add any further financial burden to the patients as well. According to our results, ALB-RDW score is associated with the prognosis of sCAP. In addition, when combined with CURB-65 score, the ability of predict the prognosis of sCAP is even stronger.

The present study had some limitations. First, this study was a retrospective study conducted in a single institution and some patients with sCAP were not contained because they admitted in other departments in our hospital or remained in the emergency observation room thus selection 
bias could not be avoided. Second, our research did not contain other inflammatory markers, such as erythrocyte sedimentation rate (ESR), procalcitonin and so on.

In conclusions, we demonstrated that ALB-RDW score was strongly associated with the prognosis of hospitalized sCAP patients especially when combined with CURB-65.

\section{Acknowledgments}

Funding: None.

\section{Footnote}

Conflicts of Interest: All authors have completed the ICMJE uniform disclosure form (available at http://dx.doi. org/10.21037/apm.2020.04.22). The authors have no conflicts of interest to declare.

Ethical Statement: The authors are accountable for all aspects of the work in ensuring that questions related to the accuracy or integrity of any part of the work are appropriately investigated and resolved. The study was approved by the Ethics Committee of Renji Hospital [No.2016-080k(b)].

Open Access Statement: This is an Open Access article distributed in accordance with the Creative Commons Attribution-NonCommercial-NoDerivs 4.0 International License (CC BY-NC-ND 4.0), which permits the noncommercial replication and distribution of the article with the strict proviso that no changes or edits are made and the original work is properly cited (including links to both the formal publication through the relevant DOI and the license). See: https://creativecommons.org/licenses/by-nc-nd/4.0/.

\section{References}

1. Cilloniz C, Dominedo C, Garcia-Vidal C, et al. Community-acquired pneumonia as an emergency condition. Curr Opin Crit Care 2018;24:531-9.

2. Montull B, Menendez R, Torres A, et al. Predictors of Severe Sepsis among Patients Hospitalized for Community-Acquired Pneumonia. PLoS One 2016;11:e0145929.

3. Wunderink RG, Waterer GW. Clinical practice. Community-acquired pneumonia. $\mathrm{N}$ Engl J Med 2014;370:543-51.

4. Quan TP, Fawcett NJ, Wrightson JM, et al. Increasing burden of community-acquired pneumonia leading to hospitalisation, 1998-2014. Thorax 2016;71:535-42.

5. Ostermann H, Garau J, Medina J, et al. Resource use by patients hospitalized with community-acquired pneumonia in Europe: analysis of the REACH study. BMC Pulm Med 2014;14:36.

6. Fine MJ, Auble TE, Yealy DM, et al. A prediction rule to identify low-risk patients with community-acquired pneumonia. N Engl J Med 1997;336:243-50.

7. Jones BE, Jones J, Bewick T, et al. CURB-65 pneumonia severity assessment adapted for electronic decision support. Chest 2011;140:156-63.

8. Sbiti-Rohr D, Kutz A, Christ-Crain M, et al. The National Early Warning Score (NEWS) for outcome prediction in emergency department patients with community-acquired pneumonia: results from a 6-year prospective cohort study. BMJ Open 2016;6:e011021.

9. Liu JL, Xu F, Zhou H, et al. Expanded CURB-65: a new score system predicts severity of community-acquired pneumonia with superior efficiency. Sci Rep 2016;6:22911.

10. Ranzani OT, Prina E, Menendez R, et al. New Sepsis Definition (Sepsis-3) and Community-acquired Pneumonia Mortality. A Validation and Clinical Decision-Making Study. Am J Respir Crit Care Med 2017;196:1287-97.

11. Ahn JH, Choi EY. Expanded A-DROP Score: A New Scoring System for the Prediction of Mortality in Hospitalized Patients with Community-acquired Pneumonia. Sci Rep 2018;8:14588.

12. Viasus D, Garcia-Vidal C, Simonetti A, et al. Prognostic value of serum albumin levels in hospitalized adults with community-acquired pneumonia. J Infect 2013;66:415-23.

13. Lee J, Kim K, Jo YH, et al. Severe thinness is associated with mortality in patients with community-acquired pneumonia: a prospective observational study. Am J Emerg Med 2015;33:209-13.

14. Yeon Lee S, Cha SI, Seo H, et al. Multimarker Prognostication for Hospitalized Patients with Community-acquired Pneumonia. Intern Med 2016;55:887-93.

15. Holter JC, Ueland T, Jenum PA, et al. Risk Factors for Long-Term Mortality after Hospitalization for Community-Acquired Pneumonia: A 5-Year Prospective Follow-Up Study. PLoS One 2016;11:e0148741.

16. Ito $A$, Ishida $T$, Tokumasu $H$, et al. Prognostic factors in hospitalized community-acquired pneumonia: a retrospective study of a prospective observational cohort. BMC Pulm Med 2017;17:78.

17. Miyazaki H, Nagata N, Akagi T, et al. Comprehensive 
analysis of prognostic factors in hospitalized patients with pneumonia occurring outside hospital: Serum albumin is not less important than pneumonia severity assessment scale. J Infect Chemother 2018;24:602-9.

18. Braun E, Domany E, Kenig Y, et al. Elevated red cell distribution width predicts poor outcome in young patients with community acquired pneumonia. Crit Care 2011;15:R194.

19. Lee JH, Chung HJ, Kim K, et al. Red cell distribution width as a prognostic marker in patients with communityacquired pneumonia. Am J Emerg Med 2013;31:72-9.

20. Braun E, Kheir J, Mashiach T, et al. Is elevated red cell distribution width a prognostic predictor in adult patients with community acquired pneumonia? BMC Infect Dis 2014;14:129.

21. Bello S, Fandos S, Lasierra AB, et al. Red blood cell distribution width [RDW] and long-term mortality after community-acquired pneumonia. A comparison with proadrenomedullin. Respir Med 2015;109:1193-206.

22. Naffaa ME, Mustafa M, Azzam M, et al. Serum inorganic phosphorus levels predict 30-day mortality in patients with community acquired pneumonia. BMC Infect Dis

Cite this article as: Chen $\mathrm{L}, \mathrm{Lu} \mathrm{XY}$, Zhu CQ. Prognostic value of albumin-red cell distribution width score in patients with severe community-acquired pneumonia. Ann Palliat Med 2020;9(3):759-765. doi: 10.21037/apm.2020.04.22
2015;15:332.

23. Mandell LA, Wunderink RG, Anzueto A, et al. Infectious Diseases Society of America/American Thoracic Society consensus guidelines on the management of communityacquired pneumonia in adults. Clin Infect Dis 2007;44 Suppl 2:S27-72.

24. Washio Y, Ito A, Kumagai S, et al. A model for predicting bacteremia in patients with community-acquired pneumococcal pneumonia: a retrospective observational study. BMC Pulm Med 2018;18:24.

25. Filho RR, Rocha LL, Correa TD, et al. Blood Lactate Levels Cutoff and Mortality Prediction in Sepsis-Time for a Reappraisal? a Retrospective Cohort Study. Shock 2016;46:480-5.

26. Park SC, Kim YS, Kang YA, et al. Hemoglobin and mortality in patients with COPD: a nationwide population-based cohort study. Int J Chron Obstruct Pulmon Dis 2018;13:1599-605.

27. Patel KV, Semba RD, Ferrucci L, et al. Red cell distribution width and mortality in older adults: a metaanalysis. J Gerontol A Biol Sci Med Sci 2010;65:258-65. 